\title{
Condições de saúde bucal em crianças de uma escola pública da zona sul de João
}

\author{
Pessoa - PB \\ Oral health conditions in children from a public school in the south zone of João Pessoa - PB \\ Condiciones de salud bucal em niños de um colégio público de la zona sur de João Pessoa - PB
}

Recebido: 28/09/2021 | Revisado: 04/10/2021 | Aceito: 13/10/2021 | Publicado: 15/10/2021

\author{
Gabriella Pires de Gusmão \\ ORCID: https://orcid.org/0000-0001-7634-7076 \\ Faculdade Nova Esperança, Brasil \\ E-mail: gabriellapg12@gmail.com \\ Débora Thaynnar de Brito Lima \\ ORCID: https://orcid.org/0000-0002-9432-8482 \\ Centro Universitário de João Pessoa, Brasil \\ E-mail: deborahthaynnar@hotmail.com \\ Maria Luiza Pires de Gusmão \\ ORCID: https://orcid.org/0000-0002-9796-0870 \\ Faculdade Nova Esperança, Brasil \\ E-mail: maluiza77@icloud.com \\ Bianca Oliveira Torres \\ ORCID: https://orcid.org/0000-0002-7256-2577 \\ Centro Universitário de João Pessoa, Brasil \\ E-mail: biancaotorres@yahoo.com.br \\ Mara Ilka Holanda de Medeiros Batista \\ ORCID: https://orcid.org/0000-0002-7314-0595 \\ Faculdade Nova Esperança, Brasil \\ E-mail: marailka@hotmail.com \\ Yuri Victor de Medeiros Martins \\ ORCID: https://orcid.org/0000-0002-9674-8907 \\ Faculdade Nova Esperança, Brasil \\ E-mail: yurivictormm@gmail.com
}

\begin{abstract}
Resumo
Este trabalho objetivou avaliar a condição de saúde bucal de crianças e adolescentes, verificando a prevalência de cárie dentária de acordo com sexo e idade nos estudantes de uma escola de ensino fundamental em João Pessoa-PB, utilizando os índices CPO-D e CEO-d. Tratou-se de uma pesquisa epidemiológica, descritiva, embasada em um estudo bibliográfico e pode ser caracterizada como observacional de natureza transversal, através da técnica direta intensiva, com abordagem quantitativa. Dos 412 alunos, a amostra totalizou 52 crianças entre 5 e 12 anos, sendo 31 do sexo feminino $(59,6 \%)$ e 21 do sexo masculino $(40,4 \%)$. O instrumento para coleta de dados foi uma ficha de avaliação com data do exame, idade e sexo do participante, bem como um modelo para preenchimento do odontograma. Os dados coletados foram armazenados na forma de banco de dados do programa StatisticalPackage for Social Sciences (SPSS) para Windows, versão 20.0 e analisados por meio de estatística descritiva. De acordo com os resultados, foi observado que os escolares avaliados possuem baixa prevalência de cárie dentária tanto na dentição decídua com valor médio de CEO-D de 0,84, quanto na dentição permanente com média de CPO-D de 1,04, satisfatório em relação à média nacional. Foi concluída a importância da implantação e continuidade de programas de educação, prevenção e promoção de saúde bucal voltados para esta população e evidenciada a necessidade de maior atenção odontológica, principalmente na dentição decídua.
\end{abstract}

Palavras-chave: Cárie dentária; Saúde pública; Odontopediatria.

\begin{abstract}
This study aimed to evaluate the oral health condition of children and adolescents, verifying the prevalence of dental caries according to sex and age in students from elementary school in João Pessoa-PB, using the CPO-D and CEO-d indexes. It was an epidemiological, descriptive research, based on bibliographical study and can be characterized as observational of a transversal nature, through the intensive direct technique, with a quantitative approach. Of the 412 students, the sample totaled 52 children between 5 and 12 years old, being 31 female $(59,6 \%)$ and 21 male $(40,4 \%)$. The instrument for data collection was an evaluation form with the date of the examination, age and gender of the participants, as well as a model for filling out the dental chart. The collected data were stored in the StatisticalPackage for Social Sciences (SPSS) for Windows, version 20.0 database and analyzed using descriptive statistics. According to the results, it is observed that the evaluated students have a low prevalence of tooth decay both in primary dentition with a mean CEO-D value of 0.84 , and in permanent dentition with a mean CPO-D of 1.04 , satisfactory in relation to
\end{abstract}


the national average. The importance of implementing and continuing education, prevention and oral health promotion programs aimed at this population was concluded, and the need for greater dental care, especially in primary dentition, was highlighted.

Keywords: Dental caries; Public health; Pediatric dentistry.

\section{Resumen}

Este estudio tuvo como objetivo evaluar el estado de salud bucal de niños y adolescentes, verificando la prevalencia de caries dental según sexo y edad en estudiantes de una escuela primaria en João Pessoa-PB, utilizando los índices CPO-D y CEO-d. Se trató de una investigación epidemiológica, descriptiva, basada en un estudio bibliográfico y que se puede caracterizar como observacional de carácter transversal, mediante la técnica directa intensiva, con un enfoque hcuantitativo. De los 412 estudiantes, la muestra fue de 52 niños entre 5 y 12 años, siendo 31 mujeres $(59,6 \%)$ y 21 hombres $(40,4 \%)$. El instrumento para la recolección de datos fue un formulario de evaluación con la fecha del examen, edad y sexo del participante, así como un modelo para el llenado de la ficha dental. Los datos recolectados se almacenaron en la base de datos StatisticalPackage for Social Sciences (SPSS) para Windows, versión 20.0 y se analizaron mediante estadística descriptiva. De acuerdo con los resultados, se observa que los estudiantes evaluados tienen una baja prevalencia de caries dental tanto en dentición temporal con un valor medio de CEO-D de 0,84, como en dentición permanente con un CPO-D medio de 1,04, satisfactorio en relación a el promedio nacional. Se concluyó la importancia de implementar y continuar los programas de educación, prevención y promoción de la salud bucal dirigidos a esta población y se resaltó la necesidad de una mayor atención odontológica, especialmente en la dentición temporal.

Palabras clave: Caries dental; Salud pública; Odontología pediatrica.

\section{Introdução}

A saúde bucal é essencial para manter o ser humano saudável como um todo, sendo um fator determinante para a qualidade de vida. Apesar da melhoria das condições de saúde bucal da população, a cárie dentária ainda é considerada como importante problema de saúde pública no Brasil e no mundo (Geus et al., 2013).

Os principais problemas bucais no Brasil, são a cárie dentária e a doença periodontal, pesquisas indicam que pelo menos $15 \%$ da população do país é desdentada. Uma boa parte da população brasileira não tem acesso à informação e a programas educacionais, muito menos a um tratamento odontológico. Para que esta realidade possa ser mudada é necessário conhecer as condições de saúde e necessidades de tratamento dos pacientes (Araújo, 2003).

Alguns fatores podem interferir e influenciar na condição de saúde bucal, como as características socioeconômicas, hábitos de consumo, área onde residem e/ou trabalham, acesso aos serviços de saúde (Matos, 2002).

A higienização bucal é considerada uma das melhores formas de prevenção da cárie e doenças periodontais. E o nível de instrução de higienização fala muito a respeito da condição bucal e necessidade de tratamento de cada paciente. As pesquisas epidemiológicas em saúde bucal possibilitam uma base sólida para as hipóteses das condições de saúde e necessidades de tratamento de uma população. Eles funcionam como um banco de dados de confiança para o desenvolvimento de programas de saúde destinados a trazer soluções das doenças bucais mais prevalentes na população estudada (Araújo, 2003).

As informações sobre hábitos bucais e higienização bucal podem auxiliar não só em um comportamento que irá ter resultados positivos na condição bucal, quanto na prevenção das doenças bucais ocasionadas pela falta ou a má higienização. Por isso a importância dessas informações que na maioria das vezes são negligenciadas. E isso devido a prevalência e a incidência dessas patologias que estão associadas a condições sociais, econômicas, políticas e educacionais e não apenas como resultado de interações biológicas na placa bacteriana dentária (Unfer; Saliba, 2000).

A realização desse estudo tem como finalidade avaliar as condições de saúde bucal das crianças e adolescentes na rede pública de ensino fundamental, possibilitando uma base para levantamento de hipóteses das condições de saúde bucal e as necessidades de tratamento, dessa forma avaliando a prevalência de cárie através do CPO-D e CEO-d e identificando os índices de cáries a partir do sexo e idade. Essa pesquisa é importante tanto para o levantamento desses dados epidemiológicos 
de prevalência de cárie, quanto para conhecer a realidade desses escolares e levar a eles informações sobre hábitos bucais que irá trazer um comportamento positivo para a prevenção da doença da cárie e futuras intervenções.

Essa pesquisa teve como objetivo principal avaliar a condição de saúde bucal dos escolares da Rede Pública da Escola Estadual de Ensino Fundamental Professor Tercia Bonavides Lins, no bairro Valentina de Figueiredo, na cidade de João Pessoa-Pb. Onde foram avaliados acerca da experiência de cárie dentária através dos índices CPO-D e CEO-d e a prevalência de cárie de acordo com o sexo e idade dos escolares.

\section{Metodologia}

Este estudo trata-se de uma pesquisa epidemiológica, descritiva, embasada em um estudo bibliográfico e pode ser caracterizado como observacional de natureza transversal, através da técnica direta intensiva, com abordagem quantitativa.

Por se tratar de uma pesquisa envolvendo seres humanos, serão atendidos os requisitos propostos pelo Conselho Nacional de Saúde, através da Resolução n 466/12 e o Estatuto da criança e do adolescente (ECA). A pesquisa só teve início após a aprovação pelo Comitê de Ética em Pesquisa em Seres Humanos do UNIPÊ e o recebimento da certidão de aprovação, com números de CAAE: 01440418.9.0000.5176 e parecer de número 3.093.700.

A pesquisa foi realizada em uma escola de ensino fundamental na cidade de João Pessoa, Paraíba, e teve um universo de 412 escolares. A amostra não probabilística foi selecionada por conveniência $(n=150)$, e realizada em crianças com idade de 5 a 12 anos. Os critérios de elegibilidade foram escolares devidamente matriculados na escola em estudo e que os pais tenham assinado Termo de Consentimento Livre e Esclarecido e as crianças tenham dado o assentimento para realização do exame. Foram excluídos da pesquisa os escolares que se sentiram constrangidos em serem observados e avaliados durante seus atendimentos ou que apresentaram alguma alteração motora ou cognitiva, e que foram impossibilitados de realizar alguma etapa da pesquisa.

Foi utilizado como instrumento para coleta de dados uma ficha de avaliação constando a data do exame, idade e sexo do participante da pesquisa, bem como um modelo para preenchimento do odontograma. Foram realizadas visitas semanais com agendamento prévio, para realização do exame clínico e preenchimento da ficha clínica individual. O exame foi realizado em sala da própria escola com boa iluminação natural. Os escolares foram posicionados sentados na cadeira com a cabeça um pouco inclinada para trás e foram utilizadas espátulas de madeira individuais e gazes para melhor visualização dos elementos dentários, prancheta, canetas azul e vermelha e a ficha de coleta de dados.

Durante o preenchimento do instrumento de pesquisa, os escolares puderam solicitar da pesquisadora qualquer explicação pormenorizada, no caso de não entendimento do procedimento a ser realizado. A pesquisadora estava devidamente paramentada cumprindo todas as determinações da Organização Mundial de Saúde com relação a paramentarão completa profissional, isto é, os EPIs (Equipamentos de Proteção Individual) como máscara, óculos, gorro, luva e jaleco e normas de biossegurança para atendimento odontológico foram rigorosamente respeitadas.

Quando, durante o exame, foi encontrado condição dentária que necessite de tratamento, os escolares foram orientados e encaminhados para Clínica Escola de Odontologia do Unipê ou a Unidade Básica de Saúde da área que a escola se encontra.

\section{Resultados e Discussão}

Diante do universo de 412 alunos a amostra totalizou 52 crianças entre 5 e 12 anos, sendo 31 do sexo feminino o que representa $(59,6 \%)$ e 21 do sexo masculino o que corresponde a $(40,4 \%)$, podendo observar a predominancia do sexo feminino, conforme a Tabela 1. 
Este dado corrobora os estudos de Barros e Bertoldi (2002) que afirma que de um total de 344.975 pessoas, em sua amostra havia $51 \%$ de mulheres e $49 \%$ de homens. Esta predominância feminina pode ser explicada pelo fato de que as mulheres são a maioria da população brasileira (BRASIL, 2004).

Tabela 1 - Distribuição da amostra de acordo com o sexo.

\begin{tabular}{ccc}
\hline SEXO & N & $\%$ \\
\hline Feminino & 31 & 59.6 \\
Masculino & 21 & 40.4 \\
Total & 52 & 100.0 \\
\hline Fonte: Dados da pesquisa (2019).
\end{tabular}

De acordo com a Tabela 2, a faixa etária das crianças que participaram do estudo está entre 5 e 12 anos, sendo a maioria das crianças possuindo idades de 6 anos $(n=12), 8$ anos $(n=10), 9$ anos $(n=13)$, e 10 anos $(n=8)$, isto é, 23,1\%, 19,2\%, $25 \%$ e $15,4 \%$ respectivamente, com a média de idade 8,29 , o desvio padrão de 1,851 , sendo a mínima de 5 anos e a máxima de 12 anos.

Em relação aos valores de cárie nas distintas faixas etárias, observa-se um aumento da ocorrência deste agravo no período da infância. No que diz respeito a este indicador, constata-se uma evolução favorável no país, mesmo que ainda pouco expressiva (VASCONCELOS et al., 2018).

As idades investigadas representam, em geral, um desafio para os profissionais com relação ao controle do comportamento da criança. Outro fator que contribui para este achado, diz respeito ao entendimento, por parte dos pais e educadores, de que os dentes decíduos serão substituídos, sendo, por este motivo, desvalorizados (Azevedo; Valença; Neto, 2012).

Tabela 2 - Distribuição da amostra de acordo com a idade.

\begin{tabular}{ccc}
\hline IDADE & $\mathbf{N}$ & $\mathbf{\%}$ \\
\hline $\mathbf{5}$ & 1 & 1.9 \\
$\mathbf{6}$ & 12 & 23.1 \\
$\mathbf{7}$ & 4 & 7.7 \\
$\mathbf{8}$ & 10 & 19.2 \\
$\mathbf{9}$ & 13 & 25.0 \\
$\mathbf{1 0}$ & 8 & 15.4 \\
$\mathbf{1 2}$ & 4 & 7.7 \\
Total & 52 & 100.0 \\
\hline
\end{tabular}

Fonte: Dados da pesquisa (2019).

Conforme a Tabela 3, pode-se observar a quantidade de alunos de cada ano escolar, que participaram da pesquisa, sendo a maioria do $1^{\circ}$ ano $(n=13), 3^{\circ}$ ano $(n=16)$, e $5^{\circ}$ ano $(n=11)$, ou seja, $25 \%, 30.8 \%$ e $21.2 \%$ respectivamente. 
Tabela 3 - Distribuição da amostra de acordo com o ano escolar.

\begin{tabular}{ccc}
\hline ANO ESCOLAR & $\mathbf{N}$ & $\%$ \\
\hline $\mathbf{1}$ & 13 & 25.0 \\
$\mathbf{2}$ & 4 & 7.7 \\
$\mathbf{3}$ & 16 & 30.8 \\
$\mathbf{4}$ & 8 & 15.4 \\
$\mathbf{5}$ & 11 & 21.2 \\
Total & 52 & 100.0 \\
\hline
\end{tabular}

Fonte: Dados da pesquisa (2019).

A Tabela 4, demonstra o CEO-D (Cariados, Extração-Indicada e Obturados - Dentes) das crianças avaliadas, 23 crianças apresentaram CEO-D $=0 ; 7$ crianças apresentaram CEO-D $=1 ; 8$ crianças apresentaram CEO-D = 2; 6 crianças apresentaram CEO-D $=3 ; 5$ crianças apresentaram CEO-D $=4$ e apenas 3 crianças apresentaram CEO-D = 6. Desse modo, observou-se na pesquisa que 44,2\% das crianças que participaram da pesquisa possuem dentes decíduos hígidos, $13,5 \%$ possuem CEO-D $=1 ; 15,4 \%$ possuem CEO-D $=2 ; 11,5 \%$ apresentam CEO-D $=3 ; 9,6 \%$ apresentam CEO-D = 4 e apenas $5,8 \%$ possuíam CEO-D = 6. Considerando-se todos os itens, o valor médio do índice CEO-D para a população estudada foi de 0,84 .

O resultado do CEO-D de 0,84, foi bastante inferior ao índice ceo-d da macrorregião sul, segundo o levantamento SB BRASIL 2003 (BRASIL, 2004) que foi de 2,62. No entanto, a média nacional para esse índice foi de 2,8 no SB BRASIL 2003 (BRASIL, 2004) tendo uma ligeira queda na mesma média no levantamento SB Brasil 2010 para 2,3 (BRASIL, 2011).

Diferentemente do estudo de Berti et al. (2013), foi observado um ceo-d de 2,42 no município de Cascavel - PR, porém, na região norte, que é uma região menos favorecida economicamente, e engloba o bairro Interlagos, encontraram um ceod de 3,16 .

A proporção de indivíduos livres de cárie (CEO-D/CPO-D = 0) diminui em função da idade, um fenômeno que é comum, considerando-se o caráter cumulativo dos índices utilizados. Aos 5 anos de idade, 46,6\% das crianças brasileiras estão livres de cárie na dentição decídua e, aos 12 anos, 43,5\% apresentam a mesma condição na dentição permanente (BRASIL, 2012).

No estudo de Hoffmann et al. (2006), apesar da prevalência de cárie dentária ser classificada como moderada, as metas propostas pela OMS tanto para a idade de 5 anos quanto de 12 anos em relação ao CEOD, CPOD e livres de cárie não foram alcançadas, sendo que esses escolares necessitam de uma atenção odontológica integral e resolutiva para que haja uma melhora das condições de saúde bucal desta população.

No estudo de Mello e Antunes (2004), na área rural de Itapetininga, observaram-se também elevados indicadores de prevalência de cáries aos cinco anos de idade, em níveis semelhantes aos observados em outras localidades e em áreas rurais da Paraíba, onde existia uma concentração natural de flúor na água, o CPO-D aos 12 anos era de 2,5, o que ainda não é considerado favorável.

Entretanto, no estudo de Traebert et al. (2001), afirma que o nível de cárie na cidade de Blumenau é comparável aos mais baixos registrados em todo mundo e, portanto, um dos mais baixos do Brasil, havendo uma acentuada redução na média de cárie dentária. 
Tabela 4 - Distribuição da amostra de acordo com o CEO-D.

\begin{tabular}{ccc}
\hline CEO-D & $\mathbf{N}$ & $\%$ \\
\hline 0 & 23 & 44.2 \\
1 & 7 & 13.5 \\
2 & 8 & 15.4 \\
3 & 6 & 11.5 \\
4 & 5 & 9.6 \\
6 & 3 & 5.8 \\
Total & 52 & 100.0 \\
\hline
\end{tabular}

Fonte: Dados da pesquisa (2019).

Segundo a Tabela 5, é possível observar o CPO-D (Cariados, Perdidos e Obturados - Dentes) dos escolares, onde 30 apresentaram dentes permanentes hígidos CPO-D $=0 ; 5$ apresentaram CPO-D $=1 ; 10$ escolares com o CPO-D = 2; 4 com o $\mathrm{CPO}-\mathrm{D}=3$, apenas uma apresentou $\mathrm{CPO}-\mathrm{D}=4$; uma apresentou CPO-D = 5 e uma apresentou CPO-D = 8, sendo 57,7\%, $9,6 \%, 19,2 \%, 7,7 \%, 1,9 \%, 1,9 \%, 1,9 \%$ respectivamente. Considerando-se todos os itens, o valor médio do índice CPO-D para a população estudada foi de 1,04 .

Tabela 5 - Distribuição da amostra de acordo com o CPO-D.

\begin{tabular}{ccc}
\hline CPO-D & N & \% \\
& & \\
\hline 0 & 30 & 57.7 \\
1 & 5 & 9.6 \\
2 & 10 & 19.2 \\
3 & 4 & 7.7 \\
4 & 1 & 1.9 \\
5 & 1 & 1.9 \\
8 & 1 & 1.9 \\
Total & 52 & 100.0 \\
\hline
\end{tabular}

Fonte: Dados da pesquisa (2019).

Do total de 52 crianças avaliadas, 12 crianças apresentam tanto o CEO-D quanto o CPO-D pois possuíam dentição mista. Um pouco inferior ao estudo de Moraes, Arsenian e Tucci, (2014), que das 160 crianças avaliadas com idade entre 5 e 13 anos, 120 apresentavam dentição mista.

O CPOD deste estudo foi de 1,04 apresentando-se melhor que o índice da macrorregião sul que foi de 2,31 (BRASIL, 2004), e também se mostrou inferior à média da mesma macrorregião no levantamento SB Brasil 2010 que apresentou o CPOD de 2 (BRASIL, 2011). Já no estudo de Berti et al. (2013), foi encontrado CPO-D = 1,91 no município de Cascavel - PR, enquanto na região norte, que abrange o bairro Interlagos, com um CPO-D de 1,98.

Pode-se verificar uma evolução positiva, no que diz respeito ao acometimento da cárie, podendo estar associada não somente à fluoretação das águas de abastecimento público, mas também a outros fatores, tais como: maior acesso aos serviços 
odontológicos, programas educativos e preventivos, uso de dentifrícios fluoretados e influência de programas públicos de atenção ao escolar (Vasconcelos et al., 2018).

Atualmente o abastecimento público com água fluoretada é considerado como um dos melhores meios de controle da cárie dentária, pois, se enquadra perfeitamente nos conceitos atuais do mecanismo de ação anticárie devido a seus atributos como agente promotor de saúde pública. Estratégias preventivas como a fluoretação da água de abastecimento público, a produção de dentifrícios fluoretados e programas preventivos, provocaram na última década uma diminuição considerável na prevalência de cárie dentária (Fejerskov e Kidd, 2013).

\section{Conclusão}

De acordo com os resultados do presente estudo, pode-se observar que a condição de saúde bucal dos escolares avaliados do sexo feminino e masculino com faixa etária de 5 a 12 anos, possui baixa prevalência de cárie dentária tanto na dentição decídua, quanto na dentição permanente, com resultados satisfatórios em relação à média nacional. Estes dados demonstram a importância da implantação e continuidade de programas de educação, prevenção e promoção de saúde bucal voltados para esta população e evidenciam a necessidade de maior atenção odontológica ao grupo estudado.

A sugestão de trabalhos futuros fica enquadrada na relevância da atuação profilática e no estímulo à prevenção frente à intervenção.

\section{Referências}

Antunes, J. L. F. \& Narvai, P. C. (2010). Políticas de saúde bucal no Brasil e seu impacto sobre as desigualdades em saúde. Revista Saúde Pública, São Paulo, 44(2), 360-365.

Araujo, M. V. A. (2003). Estudo das condições de saúde bucal e necessidades de tratamento em pacientes do curso de odontologia da Universidade Federal do Pará. Dissertação de Mestrado em Clínica Integrada - Faculdade de Odontologia, Universidade de São Paulo, São Paulo.

Azevedo, A. C.; Valença, A. M. G. \& Neto, E. A. L. (2012) Perfil epidemiológico da cárie dentária em escolares de 5 e 12 anos residentes no Município de Bayeux, Paraíba Arq Odontol. 48(2), 68-75.

Barros, A. J. D. \& Bertoldi, A. D. (2002) Desigualdades na utilização e no acesso a serviços odontológicos: uma avaliação em nível nacional. Rev. Ciência e Saúbarrde Coletiva., 7(4), 709-717.

Bernardes, A. F. S., Campos Filho, M. A., Nascimento, F. S. (2020). Cárie dentária: índice CPO-D na região norte conforme as metas da organização mundial da saúde. Faculdades Cathedral. Trabalho de conclusão de curso.

Berti, M. et. al. (2013). Levantamento epidemiológico de cárie dentária em escolares de 5 a 12 anos de idade do município de Cascavel - PR. Cad. Saúde Coletiva. 21(4), 403-406.

Brito, Z. R. G. (2019). Série histórica de levantamentos epidemiológicos: planejamento e organização da saúde bucal de caratinga. Sistema UMA - SUS. Universidade Federal de Minas Gerais (UFMG).

Fontelles, M. J.,et al. (2009). Metodologia da pesquisa científica: diretrizes para a elaboração de um protocolo de pesquisa.

Geus, J. L. et al. (2003). Prevalência de cárie e autopercepção da condição de saúde bucal entre crianças de escolas urbanas e rurais de Ponta Grossa - PR. Pesquisa brasileira odontopediatria clínica integrada. João Pessoa - PB, 13(1), 111-117.

Godoy, A. S. (1995). Pesquisa qualitativa - tipos fundamentais. Revista de Administração de Empresas, 35(3), $20-29$.

Luenenberg, L. N., Pires, P. S. (2020). Índice CPO-D em escolares da faixa etária de 12 anos - revisão integrativa. Repositório da Universidade do Extremo Sul Catarinense - UNESC.

Matos, D. L. et al. Bambuí Project: an evaluation of private, public and unionized dental services. Rev. Saúde Pública, São Paulo, $36(2), 237-43$.

Mello, T. R.C. \& Aantunes, J. L. F. (2004). Prevalência de cárie dentária em escolares da região rural de Itapetininga, São Paulo, Brasil. Cad. Saúde Pública, 20(3), 829-835.

Moraes, M. E. L. et al. (2002). Prevalência de cárie pelo índice CPO-D em portadores de síndrome de Down PGRO-Pós-Grad. Rev Odontol, 5(2).

Moraes, S. N. S.; Arsenian, M. B. \& Tucci, R. (20014). Avaliação clínica e utilização do índice CPO-D/“ceo-d” em crianças da Escola Municipal José Carlos Porto-Paraty/RJ. J Health Sci Inst. 32(3), 235-40.

Organização mundial da saúde (OMS). Levantamentos básicos em saúde bucal: manual de instruções. 3. ed. São Paulo: Santos, 1991. 
Research, Society and Development, v. 10, n. 13, e331101321202, 2021

(CC BY 4.0) | ISSN 2525-3409 | DOI: http://dx.doi.org/10.33448/rsd-v10i13.21202

Pereira A. S. et al. (2018). Metodologia da pesquisa científica. [free e-book]. Santa Maria/RS. Ed. UAB/NTE/UFSM.

Ribeiro, R. B. (2019). Índice CPOD e fatores associados dos moradores do bairro João XXIII no município de Caicó-RN. Dissertação de Mestrado Profissional em Educação, Trabalho e Inovação em Medicina - Escola Multicampi de Ciências Médicas, Universidade Federal do Rio Grande do Norte, Natal.

Traebert, J. L. et al. (2001). Prevalência e severidade da cárie dentária em escolares de seis e doze anos de idade* Rev Saúde Pública, 35(3), 283-8.

Unfer, B. \& Saliba, B. O. (2000). Avaliação do conhecimento popular e práticas cotidianas em saúde bucal. Rev Saúde Pública. São Paulo, $34(2), 19$.

Vasconcelos, F. G. G. et al. (2018). Evolução dos Índices CEO-D/CPO-D e de Cuidados Odontológicos em Crianças e Adolescentes com Base no SB Brasil 2003 e SB Brasil 2010. $R$ bras ci Saúde, 22(4), 333-340. 